\title{
EKF Based Trajectory Tracking and Integrity Monitoring of AIS Data
}

\author{
Department of Nautical Systems \\ German Aerospace Center (DLR) \\ Neustrelitz, Germany \\ Email: gregor.siegert@dlr.de
}

Gregor Siegert, Paweł Banyś, Cristina Sáez Martínez and Frank Heymann

\begin{abstract}
This work presents a novel approach for integrity monitoring of AIS data. Currently, the AIS is a valuable source for maritime traffic situation assessment but not suited for collision avoidance, as it is prone to failures and not capable of indicating the level of data integrity. To tackle this, an EKF was designed to track vessel trajectories, which allows for failure detection based on residual monitoring. For the latter, two methods for hypotheses testing were implemented, namely chisquared and GLR tests. In addition, the IMM framework was adopted for mixing the state estimates of two different process models, the CV and CTRV. The designed filter will be validated on behalf of simulated and real-world AIS data.

Index Terms-AIS, EKF, IMM, trajectory tracking, integrity monitoring, collision avoidance.
\end{abstract}

\section{INTRODUCTION}

Over the past two decades the maritime world was subject to an ever increasing number of traffic participants following the overall growth in seaborne trade (see [1] and [2]). Consequently, the vessels that carry the goods, e.g., bulk carriers, container ships, and others, do not only grow in pure numbers but also in size and tonnage. Considering that according to [3] roughly $80 \%$ of the global trade is seaborne, strict safety demands need to be deployed to protect not only humans and environment but also vital economic interests. To reduce the increased risk of collisions at sea due to dense traffic and limited manoeuvrability the IMO introduced the Automatic Identification System (AIS) in 2004 for vessels greater than $300 \mathrm{GRT}$. Equipped with an AIS transceiver a vessel will not only broadcast its own Position, Navigation and Time (PNT) data but also receive information of others in her vicinity. Shore-based VTS stations deploy AIS to enhance their possibilities of maritime surveillance. However, although the implementation of AIS has already depleted the collisions at sea, the system itself is still subject to several limitations. Long-term analyses in [4] of real-world AIS data show, that many AIS messages contain either implausible position coordinates, single parameters being set to default values, or that succeeding AIS data frames heavily violate the specified update rate. Additionally, AIS parameters such as course over ground (COG) or speed over ground (SOG) may be derived from faulty position estimates constituting to a false picture of the dynamic state of the vessel. Typically, parameters such as accuracy, integrity, continuity, and availability are used to quantify the performance of a navigation system. The AIS, however, was originally designed as radio broadcast channel for exchange of static and navigation data only. Thus, it does not provide any integrity information, nor does it ensure continuity of the service. For that reason, AIS can be currently understood as a supportive but not reliable system for maritime traffic situation assessment. In fact, the sole use of AIS for collision avoidance is illegal with respect to [5]. To compensate for the addressed drawbacks of the AIS it is proposed to perform trajectory tracking and integrity monitoring of AIS data. In this context, integrity is understood as plain measure if the current AIS data can be trusted or not. While existing methods for AIS integrity monitoring in [4] work fairly well for vessels proceeding along a straight path, they suffer from false alarms in non-linear situations (e.g. a turn manoeuvre). For that reason, this work presents a novel approach for AIS integrity monitoring and trajectory tracking based on the design of an Extended Kalman Filter (EKF). Two motion models will be introduced, that are expected to represent the vessels dynamics either in nearly straight line motion or turn manoeuvres, respectively. To merge the benefits of both models in one filter, the Interacting Multiple Model (IMM) framework of [6] was adopted, which showed good performance for vessel tracking in [7] and [8]. By monitoring the innovation of the filter, hypotheses tests can be applied to detect abnormal system behaviour. Common approaches involving the chi-squared or Generalized Likelihood Ratio (GLR) test can be found in many failure detection schemes for dynamic systems, e.g. for GNSS based applications (see [9], [10] and [11]).

The remainder of this document is structured as follows. In Section II the AIS data sources relevant to this work are introduced. The design of the filter will be presented in Section III, deriving also means for failure detection. The proposed algorithm will be validated in Section IV with respect to simulated and real-world data. The conclusion to this work is given in Section V.

\section{AIS DATA SOURCES}

The typical AIS data set contains numerous static and dynamic parameters, that are distributed over different AIS 
Table I: Dynamic parameters contained in AIS message types.

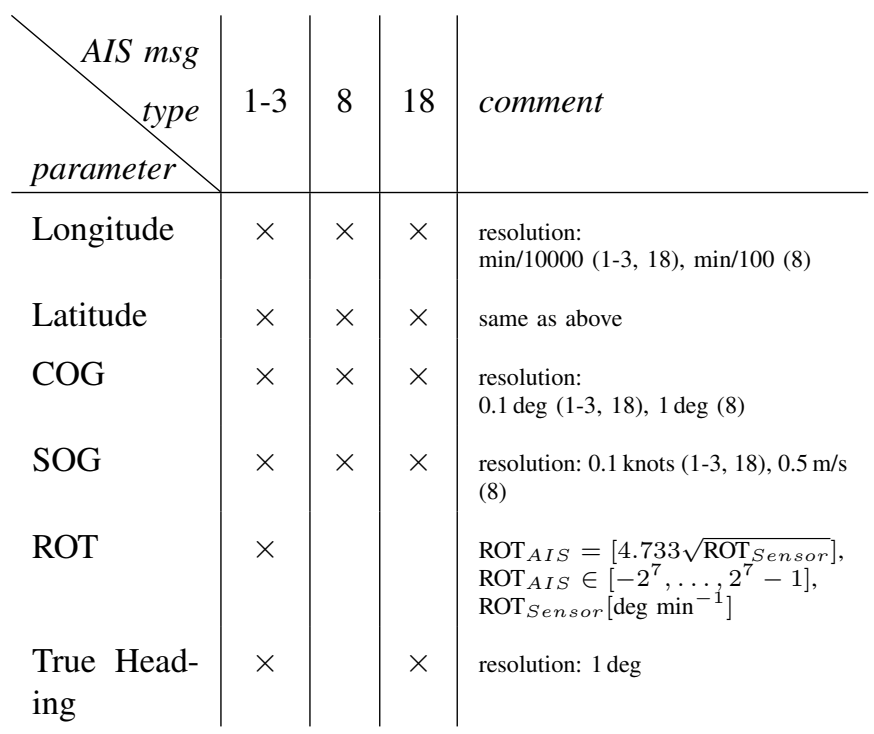

message types and specified in the International Telecommunication Union (ITU)-R recommendation [12]. Table I lists the dynamic parameters transmitted in AIS message IDs 1-3, $8^{1}$ and $18^{2}$. An additional challenge to tracking AIS trajectories is the rather slow and non-constant update rate of the messages listed in Table I. The specified time intervals between successive messages range from $2 \mathrm{~s}$ to $180 \mathrm{~s}$, depending on the dynamic state of the vessel [12]. As was shown in [4] these reporting rates are violated in a considerable amount of cases, where the transmission or reception of single or successive messages is failing.

It has to be noted that the true heading and rate of turn (ROT) parameters are typically derived from a gyro compass, whereas the position coordinates and subsequent parameters like SOG and COG depend on the output of an attached Global Navigation Satellite System (GNSS) receiver. To stay consistent with the range of possible AIS position reports the longitude, latitude coordinates as well as the SOG and COG were identified as a minimum set of parameters to describe the trajectory of a vessel. Under this premise, the vessel dynamics is modelled by two different process models that will be defined in Section III.

\section{A. AIS measurement data}

Despite a massive volume of recorded real-world AIS data available at DLR a dedicated maritime measurement campaign was conducted in October 2015 for validating the designed filter. An offshore supply vessel ${ }^{3}$ was conducting manoeuvres similar to sea trials for two successive days in the Baltic Sea (see Fig. 1). The transmitted AIS messages of the measurement

\footnotetext{
${ }^{1}$ AIS message ID 8 is a broadcast message with unspecified binary payload. The subtype with indicators DAC $=001$ and FID $=21$ contains a weather observation report and vessel parameters.

${ }^{2}$ AIS Class B transceivers issue a reduced position report.

${ }^{3}$ The BALTIC TAUCHER II was used, see http://www.marinetraffic.com/ en/photos/of/ships/shipid:134683/\#forward
}

vessel were recorded at a shore-based AIS station at the Darßer Ort Lighthouse, Germany ${ }^{4}$. In order to obtain a pseudo reference trajectory the ship was equipped with a PNT unit, that is capable of providing highly accurate position data on behalf of sensor fusion (i.e., fusion of GNSS, inertial and other onboard sensors) [13]. For this work, the reference position data was obtained from a Precise Point Positioning (PPP) solution using GPS and GLONASS observations. From this assumed ground truth of position values the associated COG and SOG of the measurement vessel were computed for later reference. For the validation of the proposed filter in Section

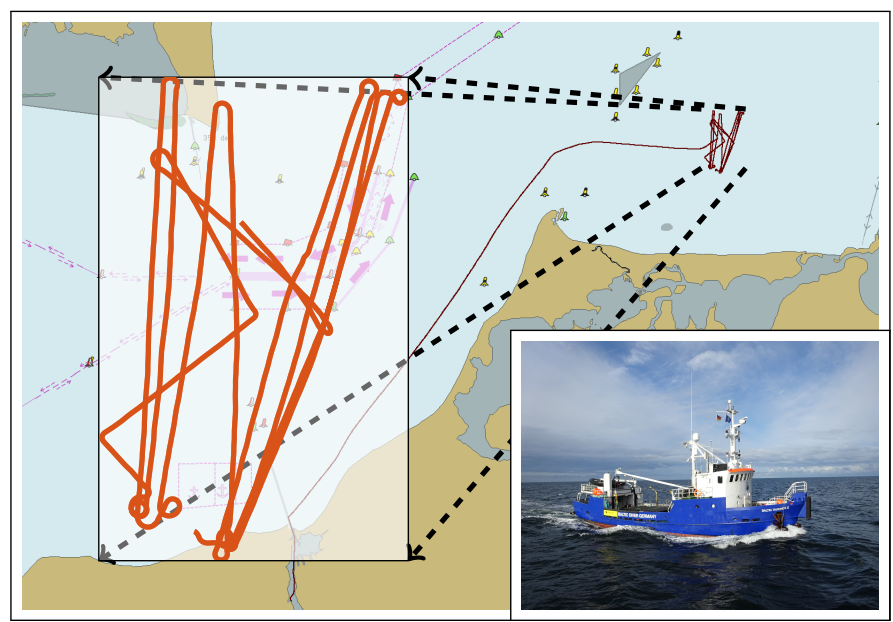

Figure 1: Nautical chart depicting the area of the measurement campaign at the Baltic Sea, zooming into the selected test trajectory. The bottom right picture shows the measurement vessel.

IV, only parts of the second day will be evaluated. Originally, a total of $15.43 \mathrm{~h}$ with reference and AIS data being recorded in parallel was available for that day. From this sequence, the subset highlighted in Fig. 1 was selected, covering $4.35 \mathrm{~h}$ or 2001 valid AIS messages, respectively.

\section{SYSTEM ARCHITECTURE}

The purpose of this work is the design of a filter that is providing continuous and plausibility-proved position data of a vessel's trajectory. Both goals can be accomplished by applying a recursive Bayesian estimator to the input AIS data. In Bayes' theorem the posterior probability of a system to be in the estimated state is based on a given prior probability and the conditioning on actual measurements [14]. A powerful implementation of the Bayesian framework is the Kalman Filter (KF) [15], which consists of an iterative prediction-correction process. The classical approach to derive integrity information based on Kalman filtering incorporates the monitoring of the residual, i.e., the innovation of the filter. In case the system dynamics can be modelled as a linear process and the GaussMarkov assumption holds, the KF also provides the optimal

${ }^{4}$ Courtesy of German Federal Waterways and Shipping Administration (WSV) 
state estimation (in terms of the minimized mean-squared error (MMSE) criterion, see [16]). The state of the system at time instance $k$ is captured in a vector $\mathbf{x}_{k}$, with $N$ state elements. Assuming a linear system model, the process model to propagate the state vector $\mathbf{x}_{k-1}$ to the next time frame can be formulated as

$$
\mathbf{x}_{k \mid k-1}=\mathbf{A}_{k} \mathbf{x}_{k-1}+\mathbf{B}_{k} \mathbf{u}_{k}+\boldsymbol{\epsilon}_{q, k}
$$

and the measurement model as

$$
\mathbf{z}_{k \mid k-1}=\mathbf{H}_{k} \mathbf{x}_{k \mid k-1}+\boldsymbol{\epsilon}_{r, k},
$$

with $\mathbf{u}_{k} \in \mathbb{R}^{L \times 1}$ being the control input to the system, $\mathbf{z}_{k \mid k-1} \in \mathbb{R}^{M \times 1}$ the measurement at time instance $k$, with respect to the previous state at $k-1$. The additive noise in the process and measurement domain is modelled by $\boldsymbol{\epsilon}_{q, k} \sim \mathcal{N}\left(\mathbf{0}, \Sigma=\mathbf{Q}_{k}\right)$ and $\boldsymbol{\epsilon}_{r, k} \sim \mathcal{N}\left(\mathbf{0}, \Sigma=\mathbf{R}_{k}\right)$, respectively. The square matrix $\mathbf{A}_{k}$ of rank $N$ denotes the state transition from $k-1$ to $k$, whereas $\mathbf{B}_{k} \in \mathbb{R}^{N \times L}$ maps the control vector to the state space. In the correction step, $\mathbf{H}_{k} \in \mathbb{R}^{M \times N}$ transforms the predicted state $\mathbf{x}_{k \mid k-1}$ to the measurement domain. In sequential Bayesian filtering the current system state is estimated on behalf of its probability distribution function (pdf), which is generally unknown. Thus, a KF not only estimates the mean of the state, but also its associated covariance $\mathbf{P}_{k}$, a square matrix of rank $N$. In the prediction step $\mathbf{P}_{k-1}$ is propagated to

$$
\mathbf{P}_{k \mid k-1}=\mathbf{A}_{k} \mathbf{P}_{k-1} \mathbf{A}_{k}^{T}+\mathbf{Q}_{k}
$$

while the correction yields

$$
\mathbf{P}_{k}=\left(\mathbf{I}_{N \times N}-\mathbf{K}_{k} \mathbf{H}_{k}\right) \mathbf{P}_{k \mid k-1} .
$$

In this context, the Kalman Gain $\mathbf{K}_{k} \in \mathbb{R}^{N \times M}$ is introduced, steering the impact of the measurement on the state correction. It is computed from

$$
\mathbf{K}_{k}=\mathbf{P}_{k \mid k-1} \mathbf{H}_{k}^{T} \mathbf{S}_{k}^{-1},
$$

being dependent on $\mathbf{S}_{k} \in \mathbb{R}^{M \times M}$, which is defined as

$$
\mathbf{S}_{k}=\mathbf{H}_{k} \mathbf{P}_{k \mid k-1} \mathbf{H}_{k}^{T}+\mathbf{R}_{k} .
$$

The actual correction of the state is obtained from

$$
\mathbf{x}_{k}=\mathbf{x}_{k \mid k-1}+\mathbf{K}_{k} \mathbf{y}_{k},
$$

with $\mathbf{y}_{k} \in \mathbb{R}^{M \times 1}$ denoting the residual between expected and actual measurement

$$
\mathbf{y}_{k}=\mathbf{z}_{k}-\mathbf{H}_{k} \mathbf{x}_{k \mid k-1} .
$$

\section{A. Filter design}

In the maritime domain, and many other real-world problems, the very essential requirement for a KF of having a linear model of the system dynamics is not fulfilled. A common approach to tackle this is the linearisation of the model around the current state estimate leading to the Extended Kalman Filter (EKF). In the following, two models for vessel motion at sea will be introduced, that constitute to an EKF-based estimation framework.

1) Constant Velocity process model: Frequently used to describe the dynamics of vessels at sea is the Constant Velocity (CV) model, e.g. in [7] or [8]. In this work, the state of the vessel for the $\mathrm{CV}$ at time instance $k$ is defined as follows:

$$
\mathbf{x}_{k}=\left[p_{x, k}, p_{y, k}, \psi_{k}, v_{k}\right]^{T}
$$

with $p_{x, k}$ and $p_{y, k}$ as position estimates in the Cartesian plane, $\psi_{k}$ as course over ground (COG) and $v_{k}$ as speed over ground (SOG). The time discrete motion model is defined as follows

$$
\begin{aligned}
p_{x, k} & =p_{x, k-1}+\Delta t v_{x, k} \\
p_{y, k} & =p_{y, k-1}+\Delta t v_{y, k} \\
\psi_{k} & =\psi_{k-1}+\Delta t \epsilon_{\psi, k} \\
v_{k} & =v_{k-1}+\Delta t \epsilon_{v, k} .
\end{aligned}
$$

Since only the absolute value for $v_{k}$ is known, the velocity components in $\mathrm{x}$ and $\mathrm{y}$ direction from (10) and (11) are defined as

$$
v_{x, k}=v_{k} \sin \psi_{k}
$$

and

$$
v_{y, k}=v_{k} \cos \psi_{k}
$$

respectively. Equations (14) and (15) couple the tracked state parameters $v_{k}(\mathrm{SOG})$ and $\psi_{k}(\mathrm{COG})$ with the position estimates. At time instance $k$, however, the best estimates for $\psi_{k}$ and $v_{k}$ are defined in (12) and (13). This leads eventually to

$$
\begin{aligned}
p_{x, k}= & p_{x, k-1}+\Delta t\left[\left(v_{k-1}+\Delta t \epsilon_{v, k}\right)\right. \\
& \left.\sin \left(\psi_{k-1}+\Delta t \epsilon_{\psi, k}\right)\right]
\end{aligned}
$$

and

$$
\begin{aligned}
p_{y, k}= & p_{y, k-1}+\Delta t\left[\left(v_{k-1}+\Delta t \epsilon_{v, k}\right)\right. \\
& \left.\cos \left(\psi_{k-1}+\Delta t \epsilon_{\psi, k}\right)\right] .
\end{aligned}
$$

2) Constant Turn Rate Velocity process model: In general, the Constant Turn Rate Velocity (CTRV) can be seen as an extension of the CV. At time instance $k$ the system state in the CTRV is defined as

$$
\mathbf{x}_{k}=\left[p_{x, k}, p_{y, k}, \psi_{k}, v_{k}, \omega_{k}\right]^{T}
$$

with the turn rate $\omega_{k}$ as additional parameter. The expression in (12) is modified to

$$
\psi_{k}=\psi_{k-1}+\Delta t\left(\omega_{k-1}+\Delta t \epsilon_{\omega, k}\right),
$$

with

$$
\omega_{k}=\omega_{k-1}+\Delta t \epsilon_{\omega, k},
$$

applying a constant velocity and turn rate model for $v_{k}$ and $\omega_{k}$. Following the argumentation from the CV process model, the position estimates for the CTRV model are now expressed as

$$
\begin{aligned}
p_{x, k}= & p_{x, k-1}+\Delta t\left[\left(v_{k-1}+\Delta t \epsilon_{v, k}\right) \ldots\right. \\
& \left.\sin \left(\psi_{k-1}+\Delta t\left(\omega_{k-1}+\Delta t \epsilon_{\omega, k}\right)\right)\right]
\end{aligned}
$$


and

$$
\begin{aligned}
p_{y, k}= & p_{y, k-1}+\Delta t\left[\left(v_{k-1}+\Delta t \epsilon_{v, k}\right) \ldots\right. \\
& \left.\cos \left(\psi_{k-1}+\Delta t\left(\omega_{k-1}+\Delta t \epsilon_{\omega, k}\right)\right)\right],
\end{aligned}
$$

keeping in mind that $\psi_{k}$ is also coupled with $\omega_{k}$.

It has to be noted that in both process models no control input $\mathbf{u}_{k}$ was defined. This is motivated by the fact, that AIS parameters that could be understood as driving force to the system, such as ROT, SOG or COG are simply insufficient in terms of consistency.

In both cases, CV and CTRV, the system state is modelled as a non-linear process, violating a key requirement of the KF. As was mentioned before, the EKF is one of the solutions to overcome this challenge (e.g., see [17]). In the EKF the process and measurement model from (1) and (2) are altered to the generalized form of

$$
\begin{aligned}
& \mathbf{x}_{k \mid k-1}=f\left(\mathbf{x}_{k-1}, \boldsymbol{\epsilon}_{q, k}\right) \\
& \mathbf{z}_{k \mid k-1}=h\left(\mathbf{x}_{k \mid k-1}\right)+\boldsymbol{\epsilon}_{r, k},
\end{aligned}
$$

where $f(\cdot)$ and $h(\cdot)$ are no more matrices, but non-linear, multi-variate functions modelling the system in the state and measurement domain, respectively. Note, that $f(\cdot)$ takes in general $\mathbf{u}_{k}$ as input argument, which is omitted in this specific case of no driving force to the system. The linearisation around the current system state is achieved by the first order Taylor series expansion, assuming that the higher order terms are negligible. The propagation of the error state covariance $\mathbf{P}_{k-1}$ from (3) is then altered to

$$
\mathbf{P}_{k \mid k-1}=\mathbf{F}_{k} \mathbf{P}_{k-1} \mathbf{F}_{k}^{T}+\mathbf{C}_{k} \mathbf{Q}_{k} \mathbf{C}_{k}^{T}
$$

and the correction of $\mathbf{P}_{k \mid k-1}$ from (4) to

$$
\mathbf{P}_{k}=\left(\mathbf{I}_{N \times N}-\mathbf{K}_{k} \mathbf{H}_{k}\right) \mathbf{P}_{k \mid k-1} .
$$

The matrices $\mathbf{F}_{k}, \mathbf{C}_{k}$ and $\mathbf{H}_{k}$ are the Jacobians of $f(\cdot)$ and $h(\cdot)$ with

$$
\begin{aligned}
\mathbf{F}_{k} & =\left.\frac{\partial f}{\partial x}\right|_{x=\mathbf{x}_{k-1}}, \\
\mathbf{C}_{k} & =\left.\frac{\partial f}{\partial x}\right|_{x=\boldsymbol{\epsilon}_{q, k}}, \\
\mathbf{H}_{k} & =\left.\frac{\partial h}{\partial x}\right|_{x=\mathbf{x}_{k \mid k-1}} .
\end{aligned}
$$

Equations (5) and (6) remain untouched, although $\mathbf{H}_{k}$ does not represent the state transition matrix to the measurement domain any more, but instead the Jacobian from (29).

3) Measurement model: In case of AIS measurements it can not be guaranteed that the measurements of COG and SOG are truly independent, as they might be derived from the position estimate. In this case the cross-correlation of these sensors needs to be reflected in the non-diagonal elements of $\mathbf{R}$, which is out of the scope of this work. The true heading, however, is obtained from a gyro compass, i.e., from an independent sensor source. But again, this parameter could not only deviate from the COG, due to sea currents or wind, some recordings have also shown corrupted true heading data. Due to these considerations, the measurement model is defined as

$$
h\left(\mathbf{x}_{k \mid k-1}\right)=\left\{\begin{array}{l}
p_{x, k \mid k-1} \\
p_{y, k \mid k-1}
\end{array} .\right.
$$

\section{B. IMM framework for model mixing}

Both process models, CV and CTRV, were introduced in the preceding paragraphs as they are expected to outperform each other in different scenarios of vessel movement. While the CV model should fit a (nearly) straight line motion best, the CTRV is assumed to be beneficial in turn manoeuvres due to the tracking of the turn rate. Although it might be tolerable to use only one model for all dynamic scenarios, it is much more appealing to merge the benefits of both in one combined filter. A computationally efficient way to do so can be found in the IMM framework, that was first introduced in [6] and proposed for vessel tracking in [8]. In contrast to other schemes that exploit the idea of multiple models running in parallel, the IMM mixes the state estimates of different models instead of yielding a hard decision between one or the other. The transition between different models (or modes) is governed by an underlying Markov process. The key concept of the IMM can be summarized in four steps:

1) Prior to each prediction-correction cycle of $D \mathrm{KFs}$, the mixed initial conditions for the $D$ state estimates $\mathbf{x}_{k-1}^{0 i}$ and their associated covariances $\mathbf{P}_{k-1}^{0 i}$ are computed. This is based on the model probabilities $p_{k-1}^{i}$, the mixing probabilities $p_{k-1}^{i \mid j}$ and the state transition probability $T_{i j}$, with $i, j \in[1, \ldots, D]$.

2) Each of the $D$ pairs $\mathbf{x}_{k-1}^{0 i}, \mathbf{P}_{k-1}^{0 i}$ is fed to a KF, which yields $\mathbf{x}_{k}^{i}, \mathbf{P}_{k}^{i}$ after a completed iteration.

3) The mode probabilities $p_{k}^{i}$ will be updated, evaluating the likelihood $\Omega_{k}^{i}$ of the model to fit the current system dynamics. The update of $\Omega_{k}^{i}$ itself is based on the innovation measure of the corresponding KF.

4) The filter output at time instance $k$ are the weighted sums of all state estimates $\mathbf{x}_{k}^{i}$ and their associated covariances $\mathbf{P}_{k}^{i}$.

The reader is referred to [6] for a detailed derivation. Special care has to be taken in the mixing stage of the IMM, in case state spaces of different dimensions are involved, which is without theoretical support from the original IMM framework. To account for this, the state vectors and error state covariances are typically augmented with zeros in places of missing elements, which will, in consequence, lead to biased mixed state estimates. In this work, an approach introduced in [18] was adopted that relies on the mutual knowledge of common and additional state elements between models of different dimensions. Prior to each mixing stage, the state estimates and their associated covariances will be adopted to obtain an unbiased state estimate of the IMM filter. 


\section{Failure detection by residual monitoring}

Besides vessel trajectory tracking, this work aims at providing a measure of the AIS data integrity. For that purpose two standard hypotheses tests were implemented, that exploit the KF property of a uniform, zero-mean, Gaussian distribution of the innovation $\mathbf{y}_{k}$ (with covariance $\mathbf{S}_{k}$ ). In fact, this assumption holds true for non-corrupted measurement data assuming the KF was designed correctly.

1) Chi-Squared test: In this case, the normalized and squared residual from (31) should follow a centralized chisquared distribution with $M$ Degrees of Freedom (DoF) (with measurement state $\mathbf{z} \in \mathcal{R}^{M \times 1}$ ).

$$
\left\|\tilde{\mathbf{y}}_{k}\right\|^{2}=\mathbf{y}_{k}^{T} \mathbf{S}_{k}^{-1} \mathbf{y}_{k}
$$

Applying the $p$-value constraint, an implausible AIS message would be detected if Equation (32) applies, with $p=1-\alpha$. This means, that the hypothesis $H_{0}\left(\left\|\tilde{\mathbf{y}}_{k}\right\|^{2}\right.$ belongs to the expected chi-squared distribution) can be rejected with probability of $p$.

$$
\left\|\tilde{\mathbf{y}}_{k}\right\|^{2}>\chi_{\alpha}^{2}
$$

A typical choice of $\alpha$ would be 0.05 , yielding a $95 \%$ certainty to either reject or accept $H_{0}$. The value $\chi_{\alpha}^{2}$ for a specific $\alpha$ and $M$ DoF can be taken from look-up tables. In order to decrease the sensitivity of the depicted approach to single outliers and in consequence the False Alarm Rate (FAR) of the system, the test is conducted for an average of $N_{\chi^{2}}$ successive innovation samples. Following the proposed method in [10], this yields a test statistic $l(k)$, with

$$
l(k)=\sum_{i=k-N_{\chi^{2}}+1}^{k} \mathbf{y}_{i}^{T} \mathbf{S}_{i}^{-1} \mathbf{y}_{i} .
$$

The constraint for issuing a failure alert from (32) is merely altered to

$$
l(k)>\chi_{\alpha}^{2}
$$

testing $l(k)$ against a chi-squared distribution of $N_{\chi^{2}} M$ DoF (in contrast to $M$ DoF in the previous case). Naturally, the choice of $N_{\chi^{2}}$, i.e., the windowing length, always results in a trade-off between a low FAR and undetected system failures. In summary, the chi-squared hypothesis test offers a simple to implement, yet limited failure detection scheme [10].

2) Generalized Likelihood Ratio Test: Compared to the chisquared method, the GLR test offers a more subtle technique to detect failures in a system by monitoring the innovation measure of a KF. In principle, the GLR is based on a common $\log$ likelihood estimator to test $H_{1}$ against $H_{0}$ under the presence of a measurement fault at time $\theta$, with $H_{0}: \mathbf{y}_{k} \sim$ $\mathcal{N}\left(\mathbf{0}, \mathbf{S}_{k}\right)$ and $H_{1}: \mathbf{y}_{k} \sim \mathcal{N}\left(\boldsymbol{\mu}, \mathbf{S}_{k}\right)$. In the context of the GLR, a system failure is mainly characterized by the change magnitude $\boldsymbol{\nu} \in \mathbb{R}^{N \times 1}$, which is typically unknown prior to the failure. The test statistic of the log likelihood estimator is then constituted from

$$
l_{k}(\theta)=\ln \left(\frac{p\left(\mathbf{y}_{k} \mid \theta, \boldsymbol{\nu}\right)}{p\left(\mathbf{y}_{k} \mid H_{0}\right)}\right) .
$$

Essentially, the GLR modifies this estimator by replacing the unknown dependencies $\boldsymbol{\nu}$ and $\theta$ with their Maximum Likelihood Estimates (MLE) $\hat{\boldsymbol{\nu}}$ and $\hat{\theta}$. This is accomplished by a double maximization of $l_{k}(\theta)$ over $\nu$ and $\theta$, following

$$
\hat{\boldsymbol{\nu}}(\theta)=\underset{\boldsymbol{\nu}}{\operatorname{argmax}} \ln \left(\frac{p\left(\mathbf{y}_{k} \mid \theta, \boldsymbol{\nu}\right)}{p\left(\mathbf{y}_{k} \mid H_{0}\right)}\right)
$$

and

$$
\hat{\theta}=\underset{\theta}{\operatorname{argmax}} \ln \left(\frac{p\left(\mathbf{y}_{k} \mid \theta, \hat{\boldsymbol{\nu}}(\theta)\right)}{p\left(\mathbf{y}_{k} \mid H_{0}\right)}\right) .
$$

Now, assuming a valid detection threshold $h$ a jump candidate $\hat{\theta}$ is accepted, if the condition

$$
l_{k}(\hat{\theta}, \hat{\boldsymbol{\nu}}(\hat{\theta}))>h
$$

is true. Still, this abstraction does not yield a methodology applicable for online detection of system failures, since the entire sample history is required to establish the test statistic in (38). For that reason a recursive formulation of the GLR was derived in [9], that allows to update the test statistic $l_{k}(\theta)$ only with respect to the latest estimate of $\boldsymbol{\nu}_{k}(\theta)$. The key idea is to model the impact of any fault in the measurements as linear regression to the corrected state estimate and to the innovation of the filter. This yields the linear regressors $\phi_{\mathbf{z}, k}^{T}$ and $\boldsymbol{\mu}_{\mathbf{z}, k}$, with the subscript $\mathbf{z}$ denoting that only faults in the measurement domain are considered. These regressors are propagated to the next time frame $k+1$ by

$$
\begin{aligned}
& \phi_{\mathbf{z}, k+1}^{T}(\theta)=\boldsymbol{\Gamma}_{\mathbf{z}}(k, \theta)-\mathbf{H}_{k} \mathbf{F}_{k} \boldsymbol{\mu}_{\mathbf{z}, k}(\theta) \\
& \boldsymbol{\mu}_{\mathbf{z}, k+1}(\theta)=\mathbf{F}_{k} \boldsymbol{\mu}_{\mathbf{z}, k}(\theta)+\mathbf{K}_{k+1} \boldsymbol{\phi}_{\mathbf{z}, k+1}^{T}(\theta),
\end{aligned}
$$

introducing a link to the EKF framework. The state transition matrix $\mathbf{F}_{k}$ is the Jacobian from Equation (27), $\mathbf{K}_{k+1}$ is the Kalman Gain from Equation (5) and $\mathbf{H}_{k}$ the state transition to the measurement domain from Equation (29). Note, that the subscripts of $k$ and $k+1$ are used in a slightly misleading manner. Here, they annotate the difference between prediction and correction step, which shall be only an exception to the previous notation in this work. The function $\boldsymbol{\Gamma}_{\mathbf{z}}(\cdot)$ in (39), however, allows us to model any expected type of fault in the observed system. For instance, a sudden jump at time $\theta$ in the measurement data would simply be represented as

$$
\boldsymbol{\Gamma}_{\mathbf{z}}(k, \theta)=\delta(k-\theta),
$$

with $\delta(\cdot)$ being the Kronecker-Delta. Following [9] and [11] the online fault detection is then obtained from a recursive least-squares framework, that yields the MLE for $\hat{\boldsymbol{\nu}}_{k}(\theta)$ to be expressed as

$$
\hat{\boldsymbol{\nu}}_{k}(\theta)=\hat{\boldsymbol{\nu}}_{k-1}(\theta)+\mathbf{L}_{k}\left(\mathbf{y}_{k}-\boldsymbol{\phi}_{k}^{T} \hat{\boldsymbol{\nu}}_{k-1}(\theta)\right),
$$

with the gain $\mathbf{L}_{k}$ and the covariance of the change magnitude $\mathbf{P}_{k}^{\nu}$ given by

$$
\mathbf{L}_{k}=\mathbf{P}_{k-1}^{\nu} \phi_{k}\left[\phi_{k}^{T} \mathbf{P}_{k-1}^{\nu} \phi_{k}+\mathbf{S}_{k}\right]^{-1}
$$

and

$$
\mathbf{P}_{k}^{\nu}=\mathbf{P}_{k-1}^{\nu}-\mathbf{L}_{k} \boldsymbol{\phi}_{k}^{T} \mathbf{P}_{k-1}^{\nu}
$$


By introducing the compact quantity

$$
\mathbf{f}_{k}(\theta)=\sum_{i=1}^{k} \phi_{i}(\theta) \mathbf{S}_{i}^{-1} \mathbf{y}_{i},
$$

the test statistic $l_{k}(\theta, \hat{\boldsymbol{\nu}}(\theta))$ can be expressed in a more convenient way as

$$
l_{k}(\theta, \hat{\boldsymbol{\nu}}(\theta))=\mathbf{f}_{k}^{T}(\theta) \hat{\boldsymbol{\nu}}_{k}(\theta) .
$$

Finally, the MLE for $\hat{\theta}$ is obtained from the value that maximizes $l_{k}(\hat{\theta}, \hat{\boldsymbol{\nu}}(\theta))$.

So far, the implementation of the depicted scheme would still yield a growing complexity during online detection with an increasing number of samples to consider. However, with the recursive framework for estimating $\hat{\boldsymbol{\nu}}$ and $\hat{\theta}$ at hand, the GLR test can be restricted to a data window of finite width $N_{G L R}$ (see [9]). This means, at any time $k$ the failure detection scheme will be utilized over the past $N_{G L R}-1$ filter states.

3) Failure detection in IMM filter: While the implementation of the introduced failure detection schemes is rather straight-forward for a stand-alone EKF some adaptions are required for the IMM filter. Due to the mixing of multiple models with different state spaces it is not possible to implement one GLR test for the combined state estimate. Instead of this, each model requires its own GLR test, that is based on the $N_{G L R}$ latest model specific Jacobians and Kalman Gains. The final decision rule is simply modified to switch between the different GLR test outputs, depending on the IMM model probability. In contrast to that, only one chisquared test is conducted for the IMM, since the required test statistic $l_{\chi^{2}}(k)$ is constructed from the residuals only. Indeed, as the measurement domain is of the same dimensionality for both models, $l_{\chi^{2}}(k)$ is built up from the past $N_{\chi^{2}}$ squared and normalized residuals from the model with the highest probability at those times.

\section{Accounting for non-continuous measurements}

To tie the integrity measure solely to the innovation monitoring related to the correction step involves the risk of underestimating the growing uncertainty of state prediction in case of missing AIS messages. The violation of the specified update rate, may it be unintentional or on purpose, occurs far more often than expected, as was shown in [4]. In these cases the error covariance $\mathbf{P}_{k \mid k-1}$ will constantly be growing, since one or more correction steps are missing in between multiple prediction cycles.

To account for this phenomenon in the integrity assessment the following method is proposed:

- Error bounds $e_{x x}$ and $e_{y y}$ shall be defined for the diagonal elements of the error covariance $\mathbf{P}_{k \mid k-1}$ that correspond to the variances of the position estimates, i.e., $\sigma_{p, x x}^{2}$ and $\sigma_{p, y y}^{2}$.

- If $\sigma_{p, x x}^{2}>e_{x x}^{2}$ or $\sigma_{p, y y}^{2}>e_{y y}^{2}$ an error flag will be raised.

- This approach yields a binary decision rule (alert - no alert)
In order to link these error bounds to physical constraints the matter of ship domains was investigated. Various researchers have made an attempt to define safety regions of vessels, based on empirical studies or surveying experienced mariners (see [19]). In the first publication addressing this issue (Fuji, in 1971, [20]) the well-known safety distance of $4 L$ in bowstern axis and $1.6 L$ abeam was defined, with $L$ being the length overall (LOA) of the current vessel. In fact, it turns out that these ship domains are commonly accepted and put to practice, as data analysis shows. For this reason, the estimated variances of the position coordinates are bounded by the Fuji constraints, that is $e_{x x}=4 L$ and $e_{y y}=1.6 L$ in y-direction of the body frame. Note, that this approach also implies different alert levels for different-sized vessels, assuming they shared the same AIS update rate and same amount of missing AIS messages. The final integrity measure at time instance $k$ is a binary indicator constituted as disjunction of $A_{F A, k}$ and $A_{B A, k}$

$$
A_{k}=A_{F A, k} \cup A_{B A, k},
$$

where $A_{F A, k}$ is the measurement failure alert based on innovation monitoring and $A_{B A, k}$ the alert for violated safety bounds based on monitoring the error state covariance.

\section{E. Algorithm}

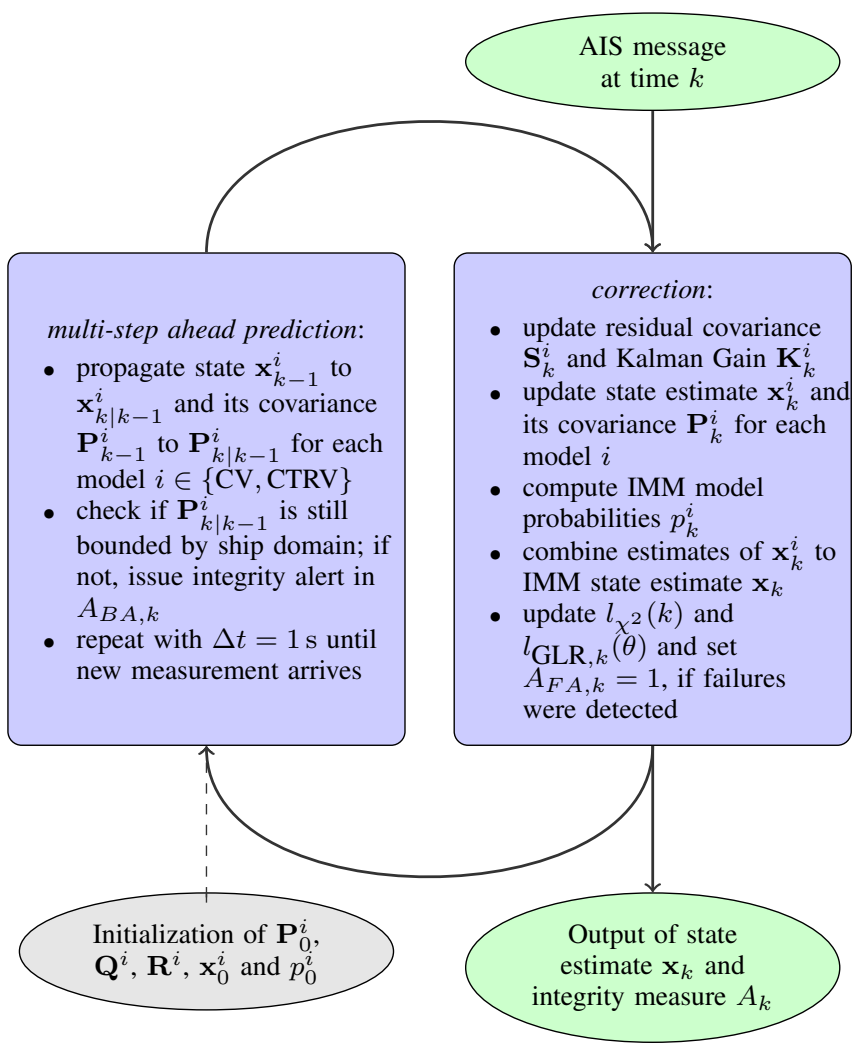

Figure 2: Sketch of proposed algorithm for trajectory tracking and integrity assessment of AIS messages based on an IMMEKF framework. 
The filter design that was introduced in this Section is summarized in Fig. 2 depicting the main steps of the algorithm. For each process model a separate EKF is embedded in an IMM framework, that mixes the initial conditions for each prediction-correction step of the individual EKFs. Unless no new AIS measurement arrives the prediction step is repeated at a rate of $\Delta t=1 \mathrm{~s}$, constantly verifying that the growing uncertainty of the predicted positions is still within bounds. After each measurement update the innovation-based test statistics for the chi-squared and GLR tests are updated for online failure detection. The output of the filter is the combined state estimate and the integrity measure, indicating whether the filtered data can be trusted or not. For the overall filter initialization the content of the first received AIS message is used to set the position, COG and SOG within $\mathbf{x}_{0}^{i}$, while the turn rate $\omega_{0}$ for the CTRV model is initialized to $0 \%$ s.

\section{RESUlts}

\section{A. Filter configuration}

Prior to the discussion of any result, the configuration of the described filter shall be listed in the following.

- The IMM incorporates a transition matrix $\mathbf{T}$, that reflects the tendency of the underlying Markov process to switch or maintain the current mode. The impact of moderate changes in different $\mathbf{T}$ is known to be rather nominal to the performance of the IMM. Thus, the transition probabilities was empirically set to

$$
\mathbf{T}=\left(\begin{array}{cc}
0.8 & 0.2 \\
0.2 & 0.8
\end{array}\right)
$$

while the initial model probabilities $p_{0}^{i}$ were equally distributed to $1 / D=0.5$.

- The EKF requires for each model the initial definition of the process and measurement covariances $\mathbf{Q}^{i}$ and $\mathbf{R}^{i}$ as well as its associated error state covariance $\mathbf{P}_{0}^{i}$. In contrast to the latter, $\mathbf{Q}^{i}$ and $\mathbf{R}^{i}$ are assumed to be time-invariant. The ratio between these two covariances is of prime importance to the performance of the EKF, as it reflects how much trust can be placed into the measurement or system model. Table II summarizes all settings for the EKF.

- To detect failures in the observed system, two methods were proposed, the chi-squared and the GLR hypotheses test. Both require the setting of only two parameters, that are $N_{\chi^{2}}$ and $\alpha=0.05$ for the former, and $N_{G L R}$ and the detection threshold $h$ for the latter. In fact, for each physical failure model expressed in $\boldsymbol{\Gamma}_{\mathbf{z}}(t, \theta)$ a different threshold might be set, since basically $\xi$ GLR tests are conducted for $\xi$ different failure models. For this work, only one type of fault is modelled, which is the jump defined in (41). This is understood as primary source of failure regarding AIS position data. A method proposed by [21] was adopted that allows to derive a reasonable value for $h_{\xi}$ in relation to a specific FAR. For that purpose, a sequence of failure-free measurement data was used. With a FAR of $10^{-3}$ a detection threshold of
Table II: Initial settings of the EKF for both implemented process models.

\begin{tabular}{l|l|l}
\multirow{2}{*}{$\mathbf{Q}$} & $\mathrm{CV}$ & $\mathrm{CTRV}$ \\
\hline \multirow{2}{*}{$\mathbf{N}$} & $\sigma_{\psi}=0.5^{\circ}$ & $\sigma_{\omega}=0.1^{\circ} / \mathrm{s}$ \\
\cline { 2 - 3 } & $\sigma_{v}=0.07 \mathrm{~m} / \mathrm{s}$ & $\sigma_{v}=0.07 \mathrm{~m} / \mathrm{s}$ \\
\hline $\mathbf{R}$ & \multicolumn{2}{|c}{$\sigma_{p, x}=\sigma_{p, y}=5 \mathrm{~m}$} \\
\hline $\mathbf{P}$ & $0.5 \times \mathbf{I}_{4 \times 4}$ & $0.5 \times \mathbf{I}_{5 \times 5}$ \\
\hline
\end{tabular}

$h_{\text {jump }}=0.67$ was obtained. Both tests are conducted over a windowed data sample of widths $N_{\chi^{2}}$ and $N_{G L R}$. However, the meaning of both parameters is somewhat different. While $N_{\chi^{2}}$ is a tuning parameter directly influencing the sensitivity of the chi-squared test, $N_{G L R}$ is also affecting the precision of the GLR failure detection. In fact, the sensitivity of the GLR method is mostly steered by the detection threshold $h_{\text {jump }}$, mentioned earlier. For this work $N_{G L R}$ and $N_{\chi^{2}}$ were empirically set to 10 and 3 , respectively.

\section{B. Simulated measurements}

A first proof of concept was conducted on behalf of simulated data to validate the principle functioning of the designed filter. The main focus was laid on the ability of the proposed schemes to detect failures in severe conditions. For that purpose, a commercial radar simulator ${ }^{5}$ was configured to simulate a test trajectory for an arbitrary outbound container vessel in the harbour of Hamburg, Germany. This radar simulator does not only produce realistic radar images, but also AIS messages, following the ITU-R specification. The simulated AIS position values, being rather ideally accurate, were artificially degraded in each component by an AWGN sequence, following $\mathcal{N}\left(0, \sigma_{A I S}=5 \mathrm{~m}\right)$. While this already yields a challenging scenario, two jumps were additionally inserted at $t_{1}=820 \mathrm{~s}$ and $t_{2}=1220 \mathrm{~s}$ in the AIS trajectory. The magnitude of the jumps was set to $m_{j}=5 \sqrt{\sigma_{x}^{2}+\sigma_{y}^{2}}=$ $5 \sqrt{2} \sigma_{A I S}$, where $m_{j}=\sqrt{\Delta p_{x}^{2}+\Delta p_{y}^{2}}$. Figure 3 depicts the simulated vessel trajectory. For verification purposes of the designed filter, three cases will be studied. The first two involve a stand-alone EKF conditioned on either the CV or CTRV process model only, omitting the mixing stages of the IMM. In the third setting, the full IMM-EKF was executed with both models running in parallel. From the results shown in Fig. 4 two main conclusions can be drawn. Firstly, all three filters show similar performance, with the IMM filter providing the best result in terms of Root Mean Square Error (RMSE) and the EKF-CV in terms of maximum 2D position error. Secondly, comparing the stand-alone performance for both process models, the CTRV is slightly worse than the $\mathrm{CV}$ for this specific test track. The latter is not surprising,

\footnotetext{
${ }^{5}$ ANS6000 by Rheinmetall Defence
} 


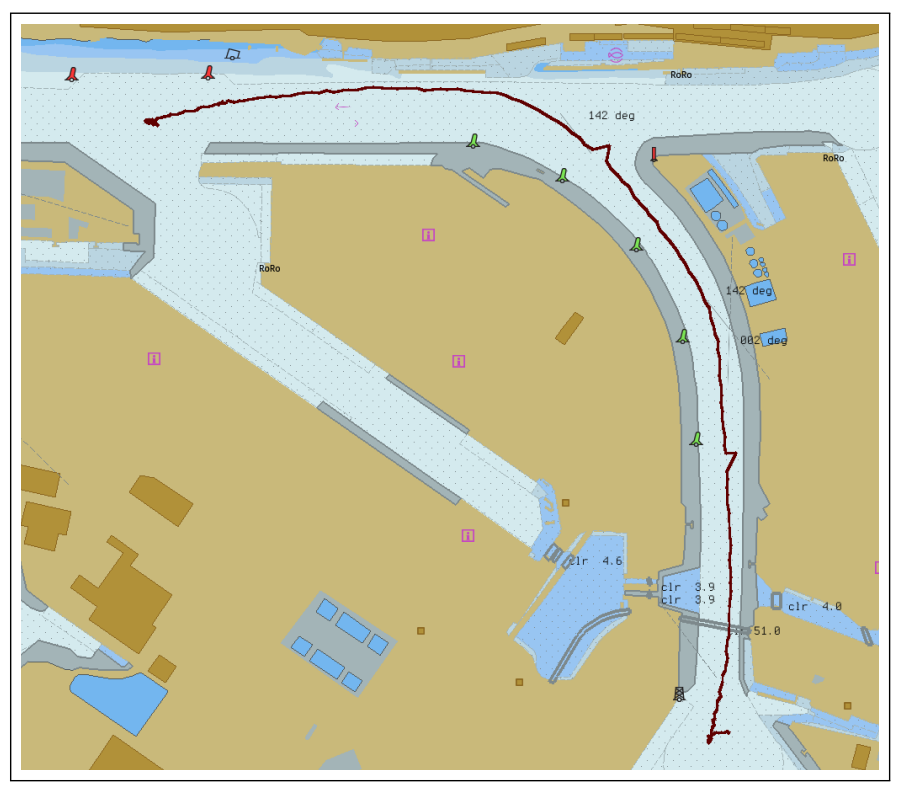

Figure 3: Nautical chart showing simulated track in the harbour of Hamburg, Germany. The inserted position faults, modelled as jumps, are clearly visible in the trajectory.
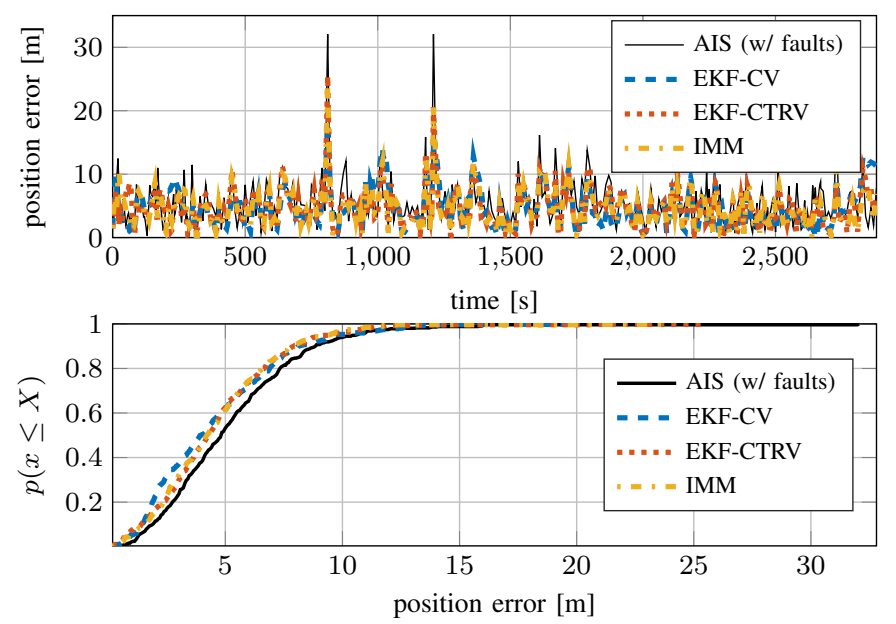

Figure 4: 2D position error of filtered and simulated AIS data, when compared to reference data (noise and fault-free). The upper plot depicts the time series of the deviations, whereas the bottom plot compares the corresponding CDFs.

since the simulated trajectory yields only a few turn manoeuvres in the first place. The principle similarity of both models is also reflected in Fig. 5 displaying the IMM-EKF model probabilities for the complete test track. During the straight path motion segments, the filter tends towards the CV model, whereas both models are just as likely during the turn manoeuvres at very low speeds. In Table III two prominent measures, namely the RMSE and the maximum deviation, for each distribution of the $2 \mathrm{D}$ position errors from Fig. 4 are listed. Another aspect of interest, is the ability of the filter to detect the artificially inserted system faults, which is
Table III: Statistics for deviation of different filter settings.

\begin{tabular}{l||l||l|l|l} 
& $\begin{array}{l}\text { AIS } \\
\text { (noisy w/ } \\
\text { faults) }\end{array}$ & $\begin{array}{l}\text { IMM- } \\
\text { EKF }\end{array}$ & EKF-CV & $\begin{array}{l}\text { EKF- } \\
\text { CTRV }\end{array}$ \\
\hline RMSE & $6.22 \mathrm{~m}$ & $5.30 \mathrm{~m}$ & $5.39 \mathrm{~m}$ & $5.39 \mathrm{~m}$ \\
\hline $\max$. & $32.08 \mathrm{~m}$ & $23.93 \mathrm{~m}$ & $19.82 \mathrm{~m}$ & $25.55 \mathrm{~m}$ \\
\hline
\end{tabular}
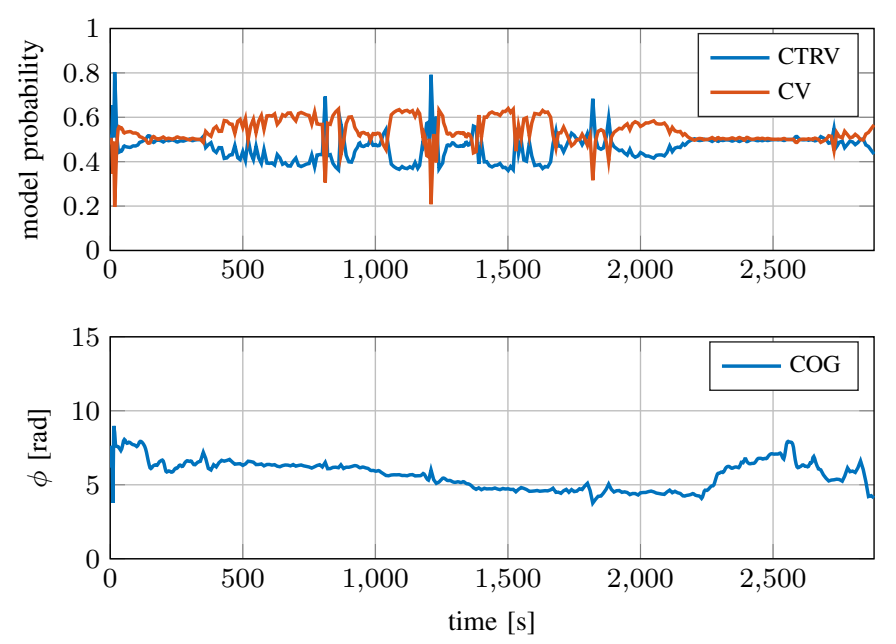

Figure 5: IMM model probabilities for simulated AIS data (upper plot) compared to the estimated COG (lower plot). The $\mathrm{CV}$ dominates the CTRV model over the entire track.

crucial for signaling an integrity alert. With the chosen settings the inserted jumps were clearly detected by both methods. However, as can be seen in Fig. 6, false alarms are issued by both test methods. For each scheme the test statistic is plotted, which is essentially compared to a fixed threshold (black, dashed line) after each measurement update. Whenever the blue curve is above this threshold an integrity alarm is raised. In contrast to the chi-squared test, the sensitivity of the GLR test could easily be adopted by raising the threshold $h_{\text {jump }}$. This illustrates the drawback of the rather trivial chi-squared test. For this particular scenario it was not possible to isolate the two failures, no matter which size of $N_{\chi^{2}}$ or $\alpha$ was chosen. It has to be stated, though, that this simulation is considered a worst-case scenario, which is assumed to be non-representative for the majority of real-world data. It is claimed in [22] that the accuracy to be expected from GPS is usually better than $3.5 \mathrm{~m}$, whereas this test track simulates an accuracy of $5 \mathrm{~m}$ at the $68 \%$ confidence interval. Thus, the detection threshold $h_{\text {jump }}$ remained deliberately unmodified, to gain a test reliable in most real-world scenarios rather than over-fitting the test to a simulation. In fact, an increased number of false alarms in troublesome environments is still acceptable, as long as serious deviations are among the detected faults. In a second, less severe scenario with $\sigma_{A I S}=3 \mathrm{~m}$ almost no false alarms are issued any more, at the cost of non-detected faults in case of chi-squared hypothesis testing (see Fig. 7). What can be seen 

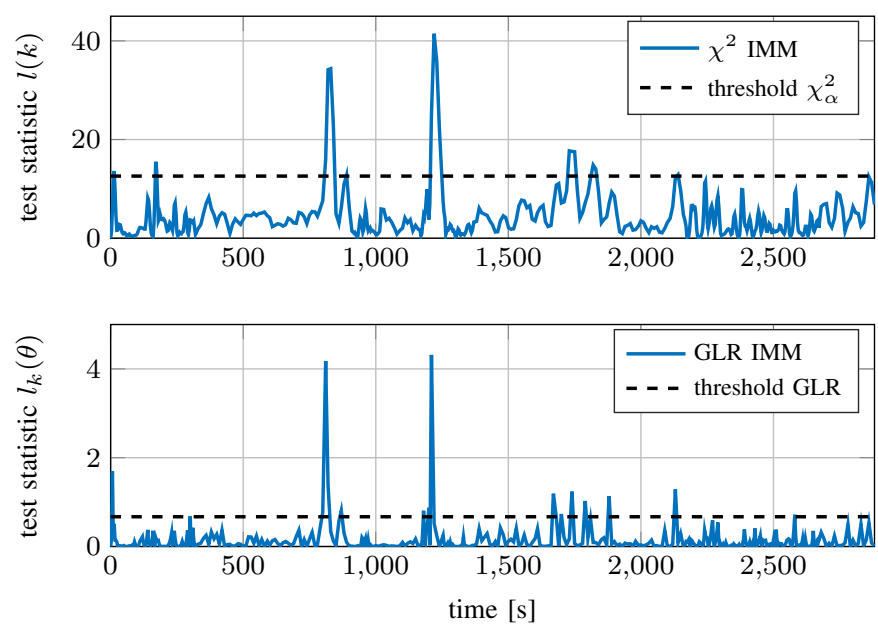

Figure 6: Test statistics for the chi-squared and GLR detection schemes showing clear peaks at the inserted measurement faults. The noisy position data $\left(\sigma_{A I S}=5 \mathrm{~m}\right)$ yields also a number of false alarms in both methods, due to the violated detection threshold (dashed line).
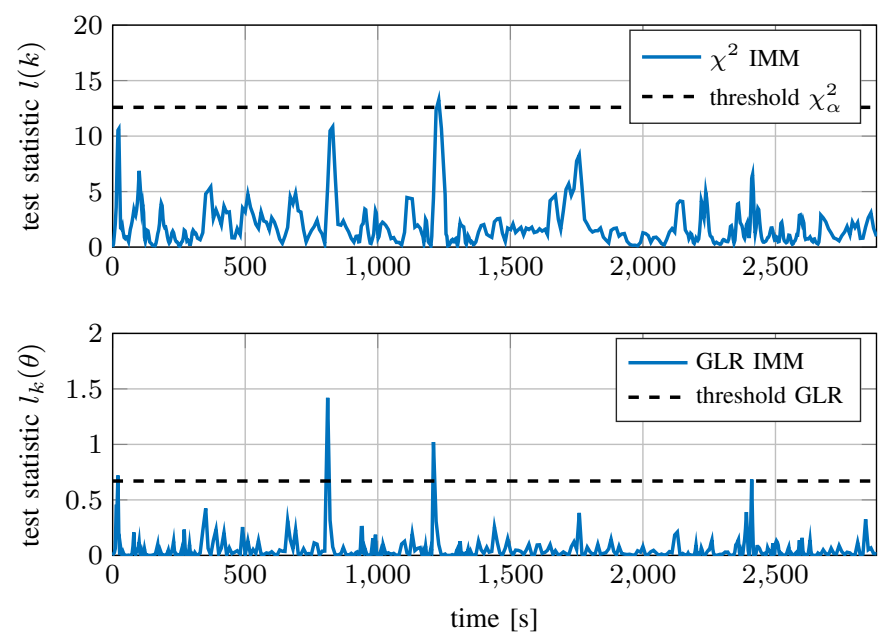

Figure 7: Failure detection in a less challenging environment $\left(\sigma_{A I S}=3 \mathrm{~m}\right)$ with less severe failures. Both methods issue almost no false alarms.

in both scenarios is the better precision of the failure detection in case of the GLR method. Due to the averaging over several samples of the squared and normalized residual, a failure in the recent past still reflects into the chi-squared test statistic at present time $k$.

\section{Real-world measurements}

Based on the measurement campaign introduced in Section II-A, the filter was also validated against real-world AIS data. As can be seen in Fig. 8, the IMM-EKF filter performs best in terms of $2 \mathrm{D}$ position error. While the stand-alone EKF conditioned on a CTRV model performs almost in the same range, the $\mathrm{CV}$ model leads to a RMSE nearly three times as bad. This is most probably due to the sharp turns of the vessel,
Table IV: Statistics for 2D position error in real-world scenario for different filter settings.

\begin{tabular}{l||l||l|l|l} 
& AIS & $\begin{array}{l}\text { IMM- } \\
\text { EKF }\end{array}$ & EKF-CV & $\begin{array}{l}\text { EKF- } \\
\text { CTRV }\end{array}$ \\
\hline RMSE & $8.10 \mathrm{~m}$ & $6.90 \mathrm{~m}$ & $18.03 \mathrm{~m}$ & $7.44 \mathrm{~m}$ \\
\hline max. & $119.90 \mathrm{~m}$ & $72.99 \mathrm{~m}$ & $87.29 \mathrm{~m}$ & $73.09 \mathrm{~m}$ \\
\hline
\end{tabular}

where the estimated COG of the CV model is adopting very slowly to the actual state of the vessel. Eventually, this leads to a misfit between measurement data and model for these time instances. In contrast to the simulation, the measurement data contains no sequences of very low speed with frequent position jumps. Indeed, the CTRV model is not falsely estimating the turn rate, which leads to a much better fit to the data. In Table IV the RMSE and the maximum of the $2 \mathrm{D}$ position error for the different filter configurations are listed.
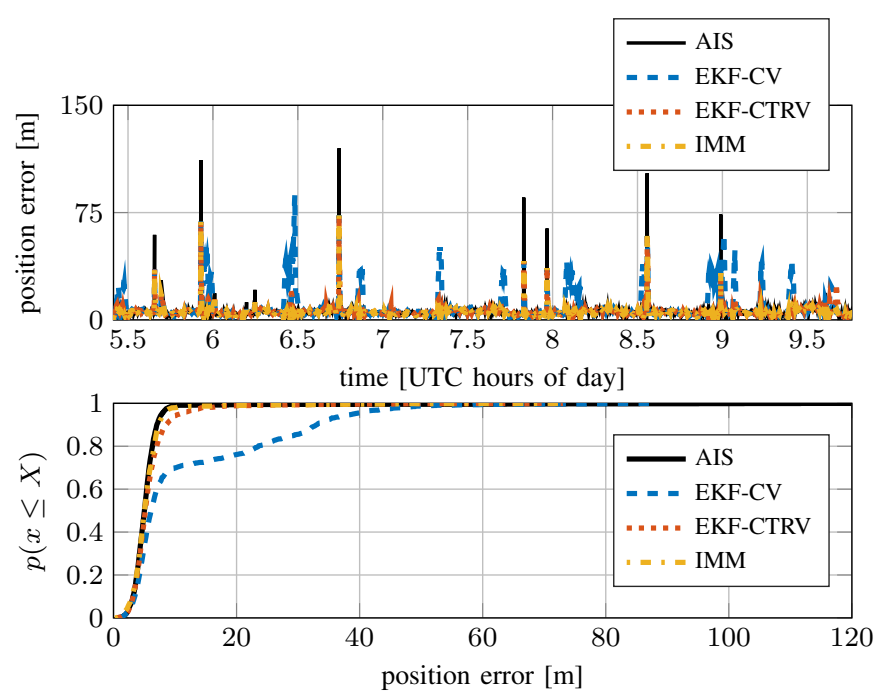

Figure 8: 2D position errors for the IMM-EKF framework, the stand-alone EKFs (conditioned on either CV or CTRV) and the original AIS data set. The deviations are compared over time in the upper and by their CDFs in the lower plot.

Regarding the model probability of the IMM filter in Fig. 9 it can be concluded that the expectations for this track are met. For visualization purposes the model probabilities are plotted against the unwrapped COG of the vessel, indicating each turn manoeuvre of the vessel. The typical behavior of an IMM filter can be very well observed, as it tends towards one particular model. In fact, the CV clearly dominates the CTRV for straight line motion parts. Even during most of the turn manoeuvres the CV is slightly more probable than the CTRV. This might be surprising considering the poor performance of the stand-alone CV model. In an IMM framework, however, the initial conditions of each prediction-correction cycle are always reset by mixing the latest state estimate and its covariances of the individual EKFs. During every turn manoeuvre the initial mixed state estimate for each separate EKF will be 

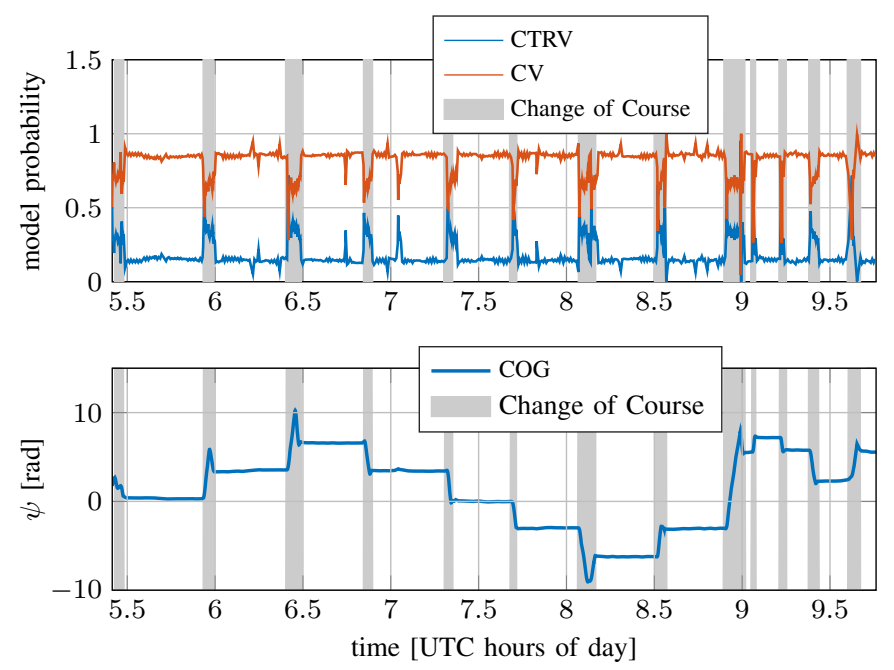

Figure 9: IMM-EKF model probabilities for the complete test track (upper plot), compared to the estimated COG (lower plot). The switching of probabilities correlates with the turn manoeuvres of the vessel, which are gray shaded.

strongly affected by the CTRV model, correcting also the COG conditioned on the CV. This yields an improved performance of the IMM filter, as it merges the benefits of both models. Thus, only the filter output can be fairly compared, not the performance of each model. Additionally to the position error of the IMM filter, the estimated state parameters COG and SOG are plotted together with their errors in Fig. 10. The impact of the AIS position errors are clearly visible in the SOG estimate, which shows heavy bumps whenever an outlier in the measurement data occurs. In these cases, the filter tries to adopt the tracked state to the offset position measurements. Again, to which extent these outliers affect the state estimate is tuned via the ratio of the process and measurement noise covariances. For the chosen settings of the IMM filter, the RMSE of the estimated COG is $4.78^{\circ}$, whereas the SOG deviates with a RMSE of $0.27 \mathrm{~m} / \mathrm{s}$ from the reference data. In contrast to the simulated track, the detected failures from the chi-squared and the GLR test are almost identical. The overall integrity alert being constituted from the expression in (47) is displayed in Fig. 11 for both methods. It can safely be stated that all faults or position errors above $21 \mathrm{~m}$ were identified as such. Both methods issue false alarms at $t \simeq 9.65 \mathrm{~h}$, which is due to a strong deviation in the position estimates (see Fig. 8). Again, the GLR proves to be more precise in failure localization, than the chi-squared test. On average, a failure was indicated for roughly $36.7 \mathrm{~s}$ in case of chi-squared, but only for $20 \mathrm{~s}$ with GLR testing. However, with this proof of concept done, for the time being it can be concluded that the chi-squared method suffices the purpose of failure detection in AIS position data.

\section{CONCLUSION}

This work introduces a novel method for integrity monitoring of maritime AIS data. An IMM-EKF framework was
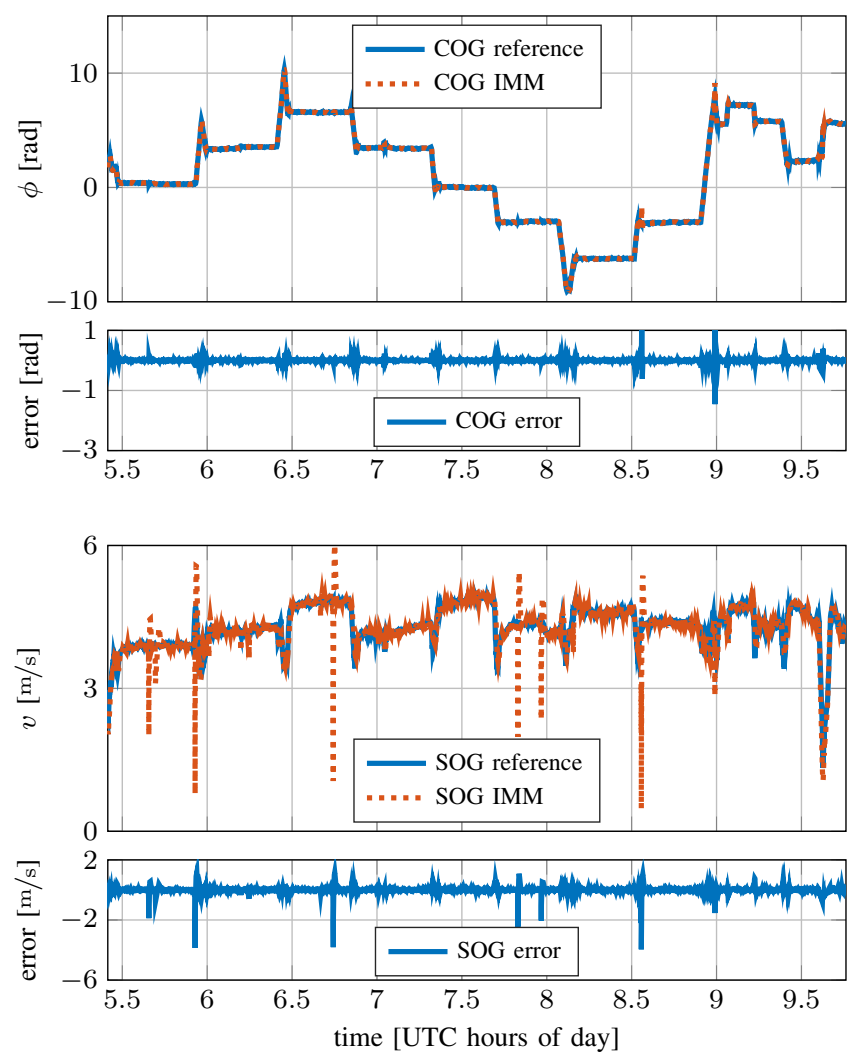

Figure 10: IMM-EKF estimates of state elements COG (upper two plots) and SOG (lower two plots) over time. Below each parameter, the error relative to the reference data is shown in a second plot.
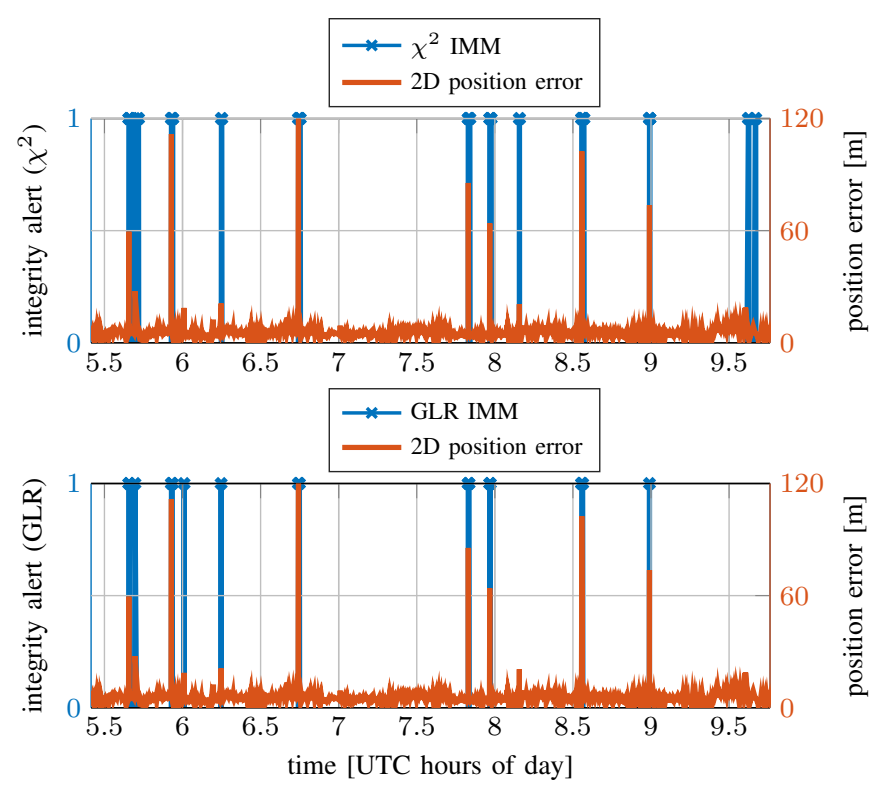

Figure 11: Final integrity measure for IMM-EKF based either on chi-squared or GLR fault detection (blue curves in both plots). Each measure is compared to the $2 \mathrm{D}$ position error of the original AIS data (red, dotted lines). 
designed, providing not only filtered and continuous PNT data of the observed vessel, but also means for integrity assessment of the transmitted data. The combination of two different process models in the IMM approach, namely the CV and CTRV, proved to be beneficial compared to a stand-alone EKF conditioned on only one of them. For failure detection the common chi-squared and GLR hypotheses tests were implemented and compared. The test scenarios comprised simulated and real-world data from a dedicated measurement campaign. It was affirmed that the CTRV model fits turn manoeuvres of the vessel best, whereas the $\mathrm{CV}$ is superior in straight line motion. In terms of failure detection, the rather trivial chisquared test was not dramatically inferior to the GLR test. All severe outliers in the AIS positions were detected as such, with a remarkably low number of false alarms in case of real-world data. However, the GLR proved to be more precise in isolating the time of failure. Additionally, if analysis showed other types of failures to be common in AIS data, the GLR could easily be extended by these failure models, due to its flexible framework. In future, comprehensive data analysis shall be conducted with the implemented filter to gain reliable numbers on AIS data integrity. Additionally, the designed framework shall be extended to fuse AIS with radar data to facilitate collision avoidance in the maritime surveillance domain.

\section{REFERENCES}

[1] United Nations Conference on Trade and Development (UNCTAD), Review of Maritime Transport 2014, ch. 2, pp. 28-48. United Nations publication, 2014.

[2] Institute of Shipping Economics and Logistics (ISL), Shipping Statistics and Market Review, vol. 56, ch. 1, pp. 5-6. ISL, 2012.

[3] United Nations, World Economic Situation and Prospects 2012, ch. 2, pp. 41-66. United Nations publication, 2012.

[4] F. Heymann, T. Noack, and P. Banyś, "Plausibility analysis of navigation related AIS parameter based on time series," in ENC, (Vienna, Austria), 2013.

[5] International Maritime Organization (IMO), "Conventions on the International Regulations for Preventing Collisions at Sea (COLREGs)," Convention, IMO, 2003. Consolidated Edition.

[6] H. A. P. Blom and Y. Bar-Shalom, "The Interacting Multiple Model Algorithm for Systems with Markovian Switching Coefficients," IEEE Transactions on Automatic Control, vol. 33, 1988.

[7] P. Braca, M. Vespe, S. Maresca, and J. Horstmann, "A Novel Approach to High Frequency Radar Ship Tracking Exploiting Aspect Diversity," Geoscience and Remote Sensing Symposium (IGARSS), 2012 IEEE International, pp. 6895 - 6898, 2012.

[8] M. Schuster, M. Blaich, and J. Reuter, "Collision Avoidance for Vessels using a Low-Cost Radar Sensor," The International Federation of Automatic Control, pp. 9673 - 9678, 2014.

[9] A. S. Willsky and H. L. Jones, "A Generalized Likelihood Ratio Approach To State Estimation in Linear Systems Subject to Abrupt Changes," IEEE Conference on Decision and Control including the 13th Symposium on Adaptive Processes, pp. 846 - 853, 1974.

[10] A. S. Willsky, "A Survey of Design Methods for Failure Detection in Dynamic Systems," Automatica, vol. 12, pp. 601-611, 1976.

[11] J. Palmqvist, "Integrity Monitoring of Integrated Satellite/Inertial Navigation Systems Using the Likelihood Ratio," in Proceedings of the 9th International Technical Meeting of the Satellite Division of The Institute of Navigation, pp. 1687-1696, ION, September 1996.

[12] ITU Radiocommunication Sector (ITU-R), "Technical characteristics for an automatic identification system using time division multiple access in the VHF maritime mobile band.," Recommendation M.1371-5, ITU, February 2014.

[13] Z. Dai, R. Ziebold, A. Born, and E. Engler, "Heading-determination using the sensor-fusion based maritime pnt unit," Position Location and Navigation Symposium (PLANS), 2012 IEEE/ION, 2012.
[14] Z. Chen, "Bayesian Filtering: From Kalman Filters to Particle Filters, and Beyond," Statistics, vol. 182, pp. 1-69, 2003.

[15] R. E. Kalman, "A new approach to linear filtering and prediction problem," Trans. ASME, vol. 82, p. 3445, 1960.

[16] C. P. Robert, The Bayesian Choice: A Decision-Theoretic Motivation. New York: Springer, 2nd ed., 2001.

[17] B. D. O. Anderson and J. B. Moore, Optimal Filtering. Prentice-Hall, Inc., 1979.

[18] J. D. Glass, W. D. Blair, and Y. Bar-Shalom, "IMM Estimators with Unbiased Mixing for Tracking Targets Performing Coordinated Turns," Proceedings IEEE Aerospace Conference, 2013.

[19] Z. Pietrzykowski and J. Uriasz, "The Ship Domain A Criterion of Navigational Safety Assessment in an Open Sea Area," The Journal of Navigation, vol. 62, pp. 93-108, 2009.

[20] Y. Fuji and K. Tanaka, "Traffic capacity," The Journal of Navigation, vol. 24, pp. 543-552, 1971.

[21] F. Gustafsson and J. Palmqvist, "Change Detection Design for Low False Alarm Rates," SAFEPROCESS'97, 1997.

[22] GPS.gov, "GPS Accuracy." http://www.gps.gov/systems/gps/ performance/accuracy/. Online; accessed 2016-01-28. 